\title{
Experimental investigations of defect sizing by transient thermography
}

\author{
by D.P. Almond, R. Hamzah, P. Delpech, Peng Wen, M.H.Beheshty and \\ M.B.Saintey
}

School of Materials Science, University of Bath, Bath, BA2 7AY, UK.

\begin{abstract}
Transient thermography images of back-drilled hole defects in bakelite sheets are shown to have spatial and temporal characteristics that are in good agreement with finite difference modelling. Defect size is found to be accurately related to image FWHM. Studies of artificial and real, impact damage, defects in carbon fibre composite plates are also presented. Good agreement is found between defect size indicated by the transient thermography and the ultrasonic c-scan techniques.
\end{abstract}

\section{Introduction}

Previous contributions [1-4] have concentrated on the analysis and modelling of the transient thermography defect imaging process and the development of a method of defect sizing. This work showed defect image formation to depend in a complex way on parameters such as: defect size; depth; severity; thermal properties and elapsed time. However, it was found that defect image size shrunk in a systematic way with time after heating (elapsed time), offering a means of defect sizing and that the initial slope of an image contrast-elapsed time graph scaled as defect depth. In this paper we present the results of: studies which have been designed to investigate the experimental validity of the modelling results and studies in which the sizing methods are being used to measure real defects in the form of impact damage of composite materials.

\section{Experimental system}

The transient thermography system used for this work was based on an Agema Thermovision 750 infrared camera whose images were recorded and digitised by a modular frame grabber TMS34020 card installed in a Viglen 486-PC. A pair of 500 Watt lamps were used to heat a sample for a period of time controlled by a second PC. The frame grabber was electronically triggered to record a sequence of thermal images after the lamps were switched off. Mechanical shutters were positioned in front of the lamps to eliminate the 'after glow' from hot lamp filaments that was found to persist for a significant period of time after they had been switched off. The camera employed an inSb detector that was sensitive to infrared radiation in the range $2-5.6 \mu \mathrm{m}$. The camera's full image scan rate was $6.25 \mathrm{~Hz}$ and these images were digitised as $256 \times 248$ pixel arrays of 8 bit numbers. The computer system recorded a set of 24 such images, sequentially or after pre-set intervals if required.

\section{Test-pieces}

Test pieces containing simulated defects of different sizes and at different depths were made by back-drilling circular flat bottomed holes in sheets of $5.8 \mathrm{~mm}$ thick bakelite. Bakelite was chosen as the sample material because of its natural black colouring and its low thermal conductivity. These properties are attractive because they ensure a high emissivity and a slow thermal transient response, compatible with the comparatively low frame rate thermal imaging system. Back-drilled hole defects of diameters $10,8,5,3$ and $2 \mathrm{~mm}$ at depths $0.5,1.2$ and 1.7 $\mathrm{mm}$ were investigated. 
A carbon fibre composite test piece containing simulated defects of differing severities was also investigated. The test piece was a 16 ply plate $29 \times 29 \mathrm{~cm}$ in which 6 artificial defects were produced by inserting $2.5 \times 2.5 \mathrm{~cm}$ squares of teflon, in place of the carbon fibres. The severities of these defects were set by the number of teflon sheets, from 2 to 12 , replacing layers of carbon fibre.

The impact damaged samples were also 16 ply composite plates which had been subjected to controlled, falling ball, impact damage in the range 1 to 5 Joules.

\section{Experimental procedures}

A sample was mounted vertically in the focal plane of the camera and heated for a short time by the lamps. The lamps were positioned to provide a fairly uniform illumination. The illumination time was determined experimentally as the time necessary to produce a 'good' defect image. Typically this was 3 seconds, for which it was estimated that peak sample surface temperature rises were below $10^{\circ} \mathrm{C}$. It should be noted that this 'long-pulse' heating method differs from the short-pulse high intensity flash excitation methods used by many other workers in this field. It has been found that good results can be obtained from bakelite and carbon fibre composite using simple modest power illumination sources operated in the long-pulse mode.

Defect images were quantified by extracting characteristic thermal profiles in the form of lines of digital data from image data files. It was found to be advantageous to average a set of four of these profiles, obtained at the same time after heating in four repeated experiments, to reduce noise to an acceptable level. Uneven heating was difficult to avoid but could be allowed for using a thermal profile obtained under the same experimental conditions from a defect-free test piece. All experimental data were treated in this way. The spatial calibration of the camera was established by imaging a mesh of known spacing placed in the same position as the samples. The modulation transfer function of the system was measured using standard methods [5]. Modulation transfer corrections, however, were found to be negligible for all the defect images obtained in this study.

\subsection{Experimental results - bakelite back-drilled hole defects}

Experimental measurements of the time dependences of the thermal contrasts and the fullwidth at half maximum (FWHM) contrast of defect image diameter obtained from $0.5 \mathrm{~mm}$ depth defects are shown in figures $1 a$ and $1 b$. The data are compared in the figure with finitedifference modelling [4] results obtained assuming bakelite to have a thermal diffusivity of $0.5 \times 10^{-6} \mathrm{~m}^{2} \mathrm{~s}^{-1}$ and including surface convection with a heat transfer coefficient of $25 \mathrm{Wm}^{-2} \mathrm{~K}^{-1}$. The latter, which is at the upper end of expected values, was found to be necessary to fit contrast at long times. The FWHMs, figure $1 b$, are in excellent agreement with the modelling predictions and provide an accurate indication of defect size for all the diameters in the range studied. The thermal contrast-time characteristics, figure 1a, of the larger defects also agree well with the modelling. Those for the two smaller defects, 3 and $2 \mathrm{~mm}$ diameters, are not included because their images could not be obtained using the same experimental conditions, camera sensitivity and heating times, as employed for the three larger defects. However, their characteristics were in close agreement to the predictions of the appropriate simulations. Note the short time range available for the FWHM measurements, figure $1 \mathrm{~b}$, corresponding to the short period of significant thermal contrast for these smaller defects.

Experimental measurements of the time dependences of the thermal contrasts and the FWHMs of $10 \mathrm{~mm}$ diameter defects at three different depths are shown in figures $2 a$ and $2 b$. Again the FWHMs, figure $2 b$, are in excellent agreement with the modelling predications, showing FWHM to provide an accurate indication of defect size irrespective of depth in this material. The data shown in figure $2 a$ has been normalised to emphasise the intrinsic differences in the time dependences of images of defects at different depths. In practice, very 
different experimental conditions had to be used to obtain the images of defects at different depths, making direct comparisons of contrast time data inappropriate. It is clear that the short time slopes of the normalised contrast time data, and simulations, vary systematically with defect depth, providing a potential means of assessing defect depth. This was noted in the earlier analytical work [1] where it was pointed out that such a measure was independent of defect size.

\subsection{Experimental results - carbon fibre composite test piece}

The thermal image of the test piece, figure 3a, reveals all six of the teflon defects, although the contrasts obtained varied considerably with the number of teflon layers. A close-up image of the 6 ply defect, figure $3 b$, was analysed quantitatively. FWHM vs time measurements of the 6 ply defect images figure 4 , indicated a defect size of $3.0 \times 3.8 \mathrm{~cm}$ rather than the expected $2.5 \times 2.5 \mathrm{~cm}$. Ultrasonic c-scan images [6] confirmed the thermographic results and showed the size of the defect to have been increased by the teflon squares slipping out of position during the curing of the composite.

\subsection{Experimental results - impact damage defects in composites}

Transient thermography and ultrasonic c-scan images of the sub-surface damage produced in a 16 ply composite by $1,2,3$ and 5 Joule impacts are shown in figure 5 . Thermographic images were taken from both the surface that was impacted and the back face of each sample. The differences between the thermographic results reflect the nature of impact damage which comprises delaminations that increase in extent between plies towards the back face These images are being used in a quantitative study of the dependence of damage on impact energy.

\section{Conclusions}

This experimental study has confirmed the potential of transient thermography as a quantitative technique for defect sizing and it has shown that the characteristics of the technique can be accurately predicted by conventional finite difference modelling. The FWHM method has been shown to determine defect size accurately in the range $2-10 \mathrm{~mm}$ at depths from 0.5 to $1.7 \mathrm{~mm}$ in bakelite. Whilst this appears to be a robust technique in this study, modelling of other materials [4] subjected to short pulse heating has indicated that FWHM fails to be related to defect size in the simple fashion found here at long times after peak contrast. The potential of the short time contrast-time curve slope for defect depth estimation has been confirmed experimentally here and it has been adopted successfully by others [7]. It is a significant improvement on earlier approaches based on peak contrast time because lateral thermal diffusion causes peak contrast time to depend on defect size. The defect depth and size determination methods used here allow for lateral thermal diffusion and are comparable to the early contrast detection method [8] which minimises these effects to provide good measures of defect depth and size

The application of the transient thermography to carbon fibre composite samples has produced encouraging results. The studies of impact damage show the technique to have adequate sensitivity and similar spatial resolution to ultrasonic c-scan. The comparisons with the established ultrasonic technique provides an important validation of the capabilities of the newer thermographic technique. However, transient thermography has the great advantages of providing images rapidly and of needing no contact with the test piece. 


\section{Acknowledgements}

The authors are grateful to: the Malaysian Government for their support of A R Hamzah; the EU for an HCM Network programme that supported P Delpech and TWI for their support of $M B$ Saintey.

\section{REFERENCES}

[1] LAU (S.K.), ALMOND (D.P.) and MILNE (J.M.). - A quantitative analysis of pulsed video thermography. NDT\&E Int.24, 1991, p. 195-202.

[2] ALMOND (D.P.) and LAU (S.K.). - Edge effects and a method of defect sizing by transient thermography., Appl.Phys. 62, 1993, p. 3569-71.

[3] ALMOND (D.P.) and LAU (S.K.). - Defect sizing by transient thermography. I: an analytical treatment. J.Phys.D: Appl.Phys. 27, 1994, p. 1063-69.

[4] SAINTEY (M.B.) and ALMOND (D.P.). - Defect sizing by transient thermography II: a numerical treatment. J.Phys.D: Appl.Phys. 28, 1995, p.2539-46.

[5] WILLIAMS (T.L.). - The MTF of thermal imaging cameras - its relevance and measurement. Proc. Quantitative Infrared Thermography (QIRT 92) Eurotherm Seminar 27, 1992, p. 51-56.

[6] HUTCHINS (D.A.) private communication.

[7] HAN (X.), FAVRO (L.D.), KUO (P.K.) and THOMAS (R.L.). - Early-time pulse echo thermal wave imaging. Rev.Proc. QNDE 15 ed. D 0 Thompson and D E Chimenti, Plenum Press, New York, 1996, p. 519-24.

[8] KRAPEZ (J.-C) and BALAGEAS (D). - Early detection of thermal contrast in pulsed stimulated infrared thermography. Proc. Quantitative Infrared Thermography (QIRT 94) Eurotherm Seminar 42, 1994, p.260-266.

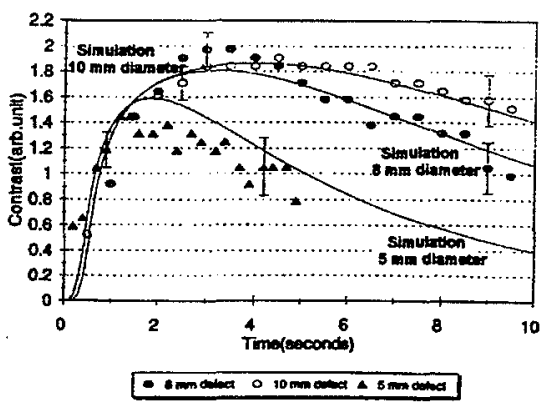

(a)

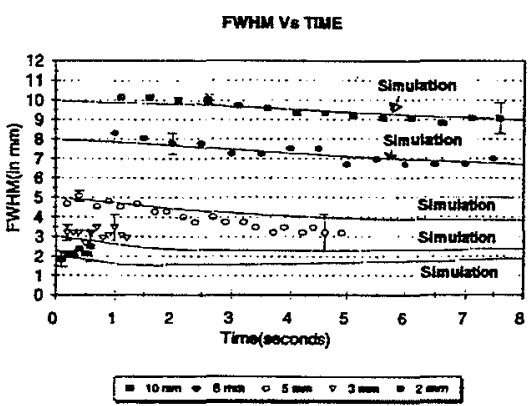

(b)

Fig. 1(a) thermal contrast and (b) FWHM measurements of back-drilled hole defect images compared with simulations. Hole depth $0.5 \mathrm{~mm}$, material bakelite, diameters shown in figure. 


\section{http://dx.doi.org/10.21611/qirt.1996.038}

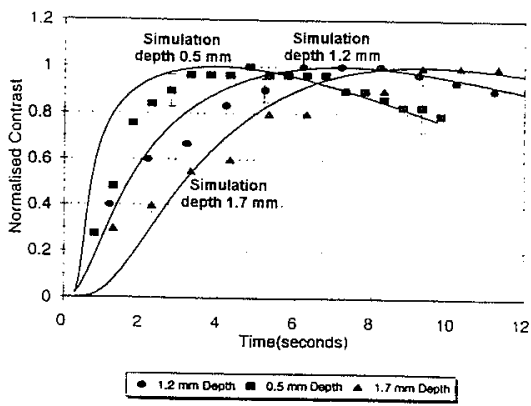

(a)

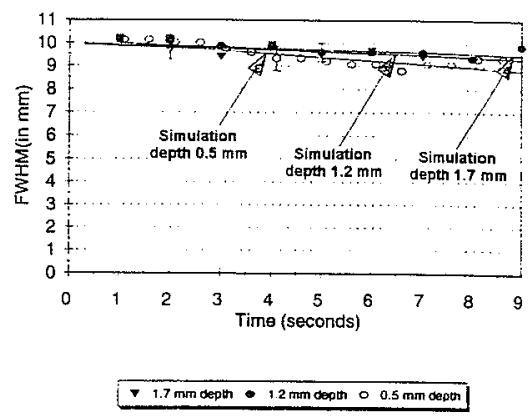

(b)

Fig. 2 (a) thermal contrast and (b) FWHM measurements of back-drilled hole defect images compared with simulations. Hole diameter $10 \mathrm{~mm}$, material bakelite, depths shown in figure.

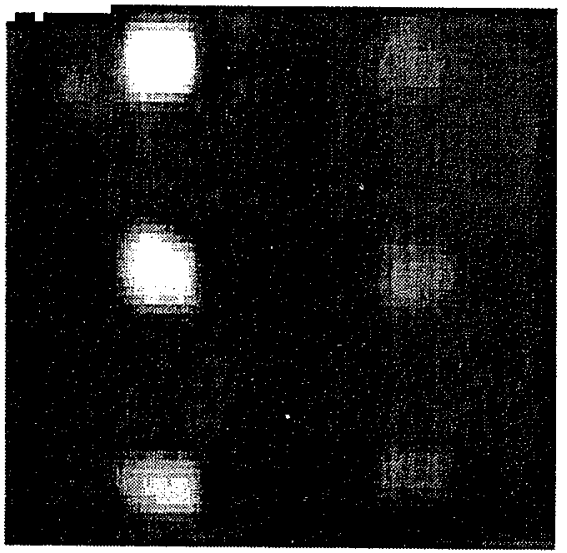

(a)

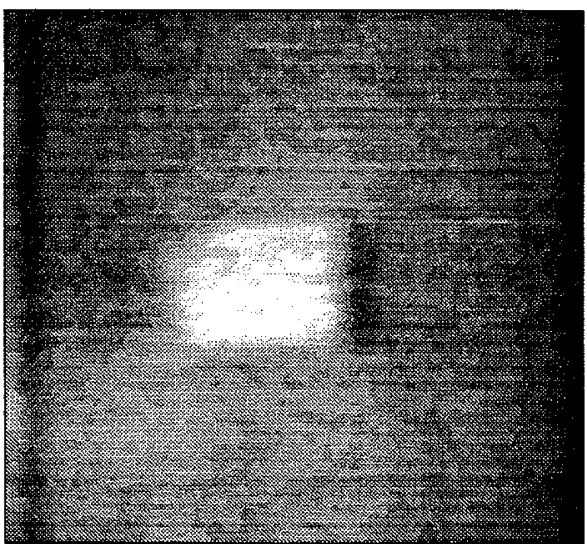

(b)

Fig. 3 (a) Transient thermography image of carbon fibre test piece containing teflon squares, $2,4,6,8,10$ and 12 ply top right clockwise to top left. (b) Close-up image of the 6 ply defect.

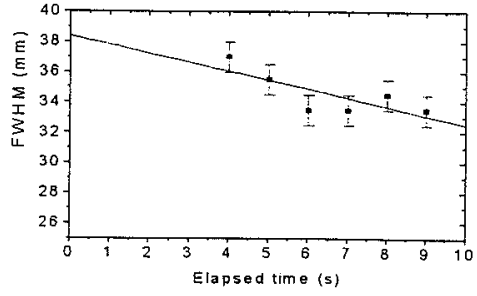

(a)

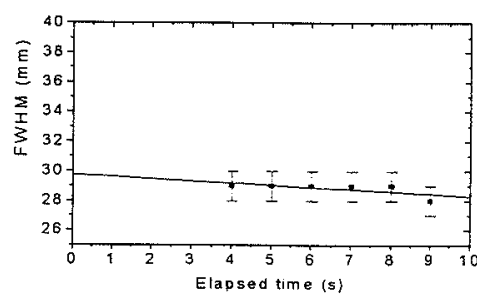

(b)

Fig. 4 FWHM vs. elapsed time image measurements of the 6 ply teflon defect in the carbon fibre test piece, (a) horizontal and (b) vertical. 


\section{http://dx.doi.org/10.21611/qirt.1996.038}
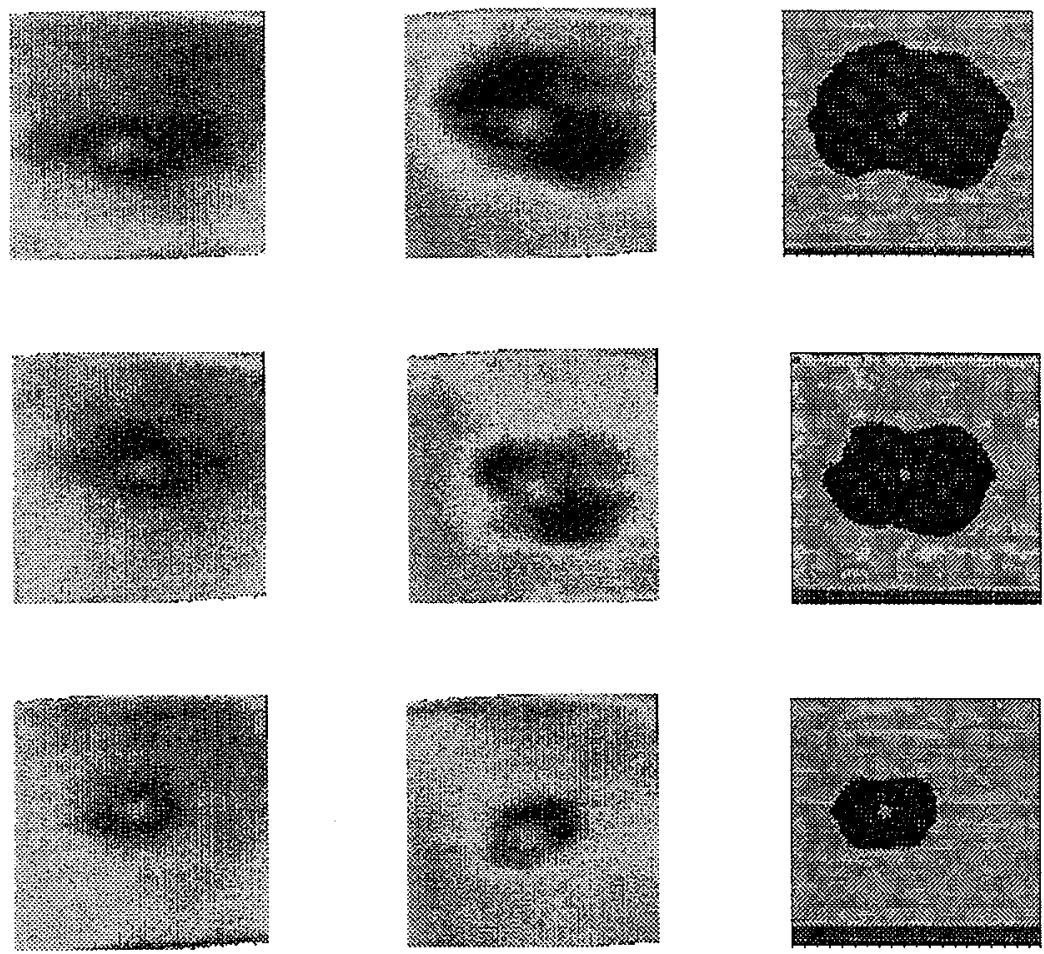

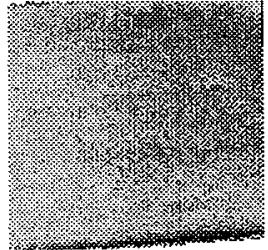

(a)

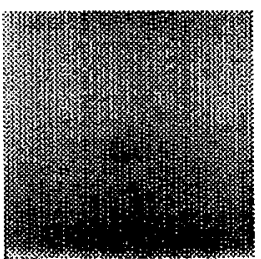

(b)

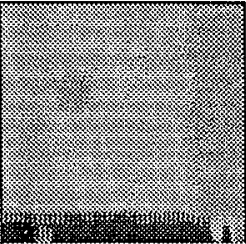

(c)

Fig. 5 Transient thermograph images of $5 \mathrm{~J}, 3 \mathrm{~J}, 2 \mathrm{~J}$ and $1 \mathrm{~J}$ impact damage, [column (a)] from impact face, [column (b)] from back face compared with ultrasonic C-scan images [column (c)]. Image area is about $40 \times 40 \mathrm{~mm}^{2}$. 